Supporting Information

\title{
A vertical flow method for sensitive Raman protein measurement in aqueous solutions
}

\author{
Shu-Chi $\mathrm{Li}^{1}$ and Hirotsugu Hiramatsu ${ }^{1,2, *}$
}

1. Department of Applied Chemistry and Institute of Molecular Science, National Chiao Tung University, Hsinchu 30010, Taiwan.

2. Center for Emergent Functional Matter Science, National Chiao Tung University, Hsinchu 30010, Taiwan.

*E-mail: hiramatu@nctu.edu.tw

Table of Contents

1. Determination of the size of focal point of the excitation laser

2. Procedure of the data analysis 


\section{Determination of the size of focal point of the excitation laser}

We examined the size of the focal point of the excitation laser. For this purpose, position of a razor was scanned along a direction perpendicular to the propagation direction of the incident laser beam at the focal point, and the intensity of laser beam reflected and scattered on the surface of the razor was recorded as a

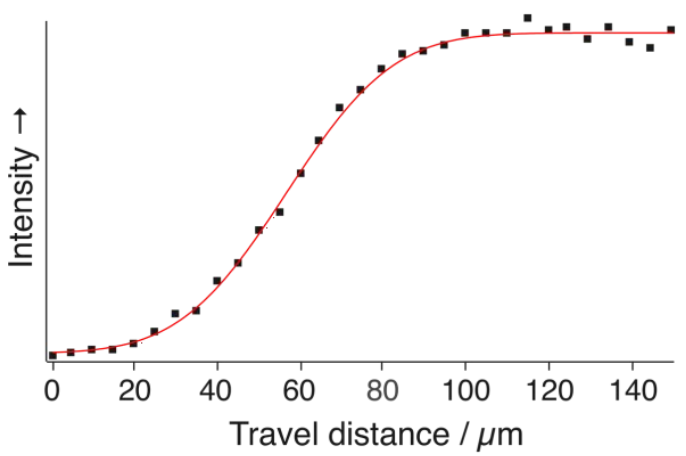

Figure S1 ( $\square)$ Intensity of laser beam reflected and scattered on the surface of the razor against travel distance of the razor. Red line shows fitted curve using the Gauss error function.

function of the travel distance of the razor.

Figure S1 shows a plot of the intensity $\left(I_{\text {laser }}\right)$ of the scattered light on the surface of a razor against the travelling distance $(d)$. Sinusoidal plot of $I_{\text {laser }}$ on $d$ was fitted successfully with the Gauss error function (Fig. S1, red). The band width, at which the intensity diminishes to $1 / e$, of the Gaussian function was determined to be $57 \mu \mathrm{m}$ (FWHM was consequently calculated to be $48 \mu \mathrm{m})$. The intensity of the laser beam at $d=50 \mu \mathrm{m}$ (at the edge of $0.1 \mathrm{~mm}$-pinhole) was $4.8 \%$ of the peak intensity. According to the calculation using the Gauss error function, less than $2 \%$ of the intensity of the incident beam was blocked at the pinhole of $100 \mu \mathrm{m}$ in diameter. 


\section{Procedure of the data analysis}

The Raman spectra of the sample solution and the solvent were recorded separately (Figure S2(a)). The difference spectrum (the solution minus the solvent multiplied by a coefficient) was calculated (Figure S2(b)); the coefficient for the subtraction was chosen such that the $1700-1800 \mathrm{~cm}^{-1}$ region of the difference spectrum became flat.

In order to evaluate the signal intensity of the Raman band of BSA, we calculated the sum of the peak height of the three bands at 1647, 1445, and $1328 \mathrm{~cm}^{-1}$. The peak height of each band was derived from the Raman intensities at the peak calculated from a baseline (Figure S2(c)). An average of 11 data points (the center \pm 5 points) was used (Table S1). The baseline was obtained as a line that connects the two points flanking the peak position, and the value of the baseline at the peak position was used as an origin to calculate the peak height.

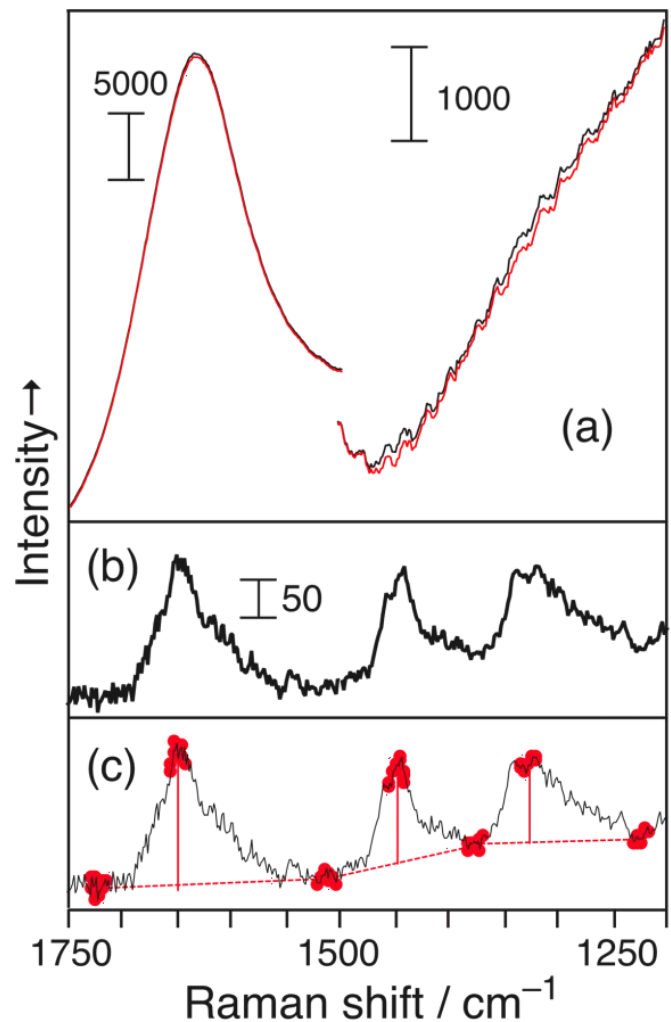

Figure S2 (a) the Raman spectra of the sample solution (black) and the solvent (red). The concentration of BSA is $0.3 \mathrm{mg} \mathrm{mL}-1$. (b) difference Raman spectrum (the sample solution minus the solvent multiplied by a coefficient). (c) Procedure to obtain the peak height of each band. See text.

\begin{tabular}{|c|c|c|}
\hline Center $/ \mathrm{cm}^{-1}$ & Range $/ \mathrm{cm}^{-1}$ & purpose \\
\hline 1742 & $1735-1749$ & baseline \\
\hline 1647 & $1640-1655$ & peak \\
\hline 1514 & $1507-1522$ & baseline \\
\hline 1445 & $1438-1453$ & peak \\
\hline 1373 & $1366-1381$ & baseline \\
\hline 1328 & $1320-1336$ & peak \\
\hline 1223 & $1215-1231$ & baseline \\
\hline
\end{tabular}

